\title{
Spatial Array Processing in a 3D Multiuser Network
}

\author{
Johanna Ketonen, Xiaojia Lu and Markku Juntti \\ Centre for Wireless Communications \\ University of Oulu, Finland \\ \{johanna.ketonen, markku.juntti\}@ee.oulu.fi
}

\begin{abstract}
The performances of spatial array processing algorithms along with three-dimensional (3D) antenna arrays are studied in this paper. Optimal linear beamforming is compared to codebook based processing. The elevation domain is further utilized when applying separate weighting vectors for the azimuth and elevation dimensions. The theoretical capacities of the beamforming systems with different antenna arrays are compared. Simulations are performed with the latest $3 \mathrm{D}$ channel models in a multiuser network. The best performance is obtained with the horizontal uniform linear arrays (ULA) when optimal beamforming is used. However, the performances of ULA and uniform planar array (UPA) are similar when codebook based beamforming is employed.
\end{abstract}

\section{INTRODUCTION}

The demand for higher spectral efficiency in wireless communication systems has been growing due to the increased data rate and quality of service requirements. One solution has been to place antennas in a three dimensional (3D) space in the base station (BS). This enables the utilization of the elevation domain in beamforming. An individual beam pattern can be assigned to each user in both elevation and azimuth domains. Field trials in [1] showed major improvements in system performance when separating the simultaneous transmissions to different users in the elevation domain.

The spatial correlation and capacity for rectangular arrays were discussed in [2]-[4]. Both the azimuth-of-arrival (AOA) and elevation-of-arrival (EOA) were shown to affect the spatial correlation of uniform rectangular arrays in [2] with the AOA having the highest impact. Two-dimensional channel models have been used extensively in multiple antenna research. Recently, those models have been extended to three-dimensional ones. The WINNER II project proposed a 3D channel model in [5] which was not based on extensive measurements. The Third Generation Partnership Project (3GPP) presented a three-dimensional model in [6] more recently.

The uniform planar arrays (UPA) and the uniform linear arrays (ULA) were compared in [7] via system level simulations. The UPA was found to perform better in interference limited scenarios but the difference between the two arrays grew smaller as the number of cooperating BSs increased. However, the geometries of the ULA and UPA were the same and the WINNER II model was used in [7]. The performance of different types of antenna arrays utilizing the latest channel models while taking into account different beamforming techniques and their complexities have not been discussed in the literature.

In this paper, the 3D channel models are utilized when comparing the performances of different beamforming techniques with different antenna arrays, namely the UPA and ULA. 3D MIMO is studied both from point-to-point and system level view. Comparisons between the ULA and UPA are first made with their spatial correlation functions. The performance is then simulated with the most recent 3D channel model and multicell network simulations. The theoretical complexity of beamforming weight calculation is then reported.

The rest of the paper is organized as follows. The system and channel models are presented in II. The spatial correlation and beamforming capacities are reported in III and the array processing algorithms are introduced in IV. Performance examples are given in $\mathrm{V}$ and complexity results are discussed in VI. Conclusions are finally drawn in VII.

\section{SyStem AND CHANNEL MODEL}

\section{A. Multicell System}

The considered cellular system consists of $K \mathrm{MSs}$ and $B$ BSs in flat fading channels. All MSs have one transmit antenna while BS $i$ has $N_{i}$ receive antennas. The received signal $\boldsymbol{r}_{i} \in$ $\mathbb{C}^{N_{i} \times 1}$ at the $i$ th $\mathrm{BS}$ is written as

$$
\boldsymbol{r}_{i}=\sum_{k=1}^{K} \sqrt{p_{k}} \boldsymbol{h}_{k, i} s_{k}+\boldsymbol{n}_{i}
$$

where $p_{k}$ is the transmit power of MS $k, s_{k}$ is the transmitted symbol of MS $k$ with average power normalized to $1, \boldsymbol{h}_{i j} \in$ $\mathbb{C}^{N_{i} \times 1}$ is the complex channel response from the $k$ th MS to the $i$ th BS and $\boldsymbol{n}_{i} \in \mathbb{C}^{N_{i} \times 1}$ is the additive white Gaussian noise (AWGN) vector at $\mathrm{BS} i$ with variance $\sigma_{i}^{2}$ for each receive antenna.

\section{B. 3D Spatial Channel}

The 3D channel model is based on the WINNER II channel model which is a geometry based stochastic model [8]. A general form of MIMO channel matrix is given by

$$
\boldsymbol{H}(t, \tau)=\sum_{n=1}^{N} \boldsymbol{H}_{n}(t ; \tau),
$$

where $n$ is the path index, $t$ is the time index, $N$ is the total number of paths, and $\tau$ is the delay time. $\boldsymbol{H}_{n}(t, \tau)$ is the channel matrix for cluster $n$ which is expressed as [8]

$$
\boldsymbol{H}_{n}(t ; \tau)=\int \boldsymbol{F}_{\mathrm{Rx}}(\phi) \boldsymbol{H}(t ; \tau, \phi, \varphi) \boldsymbol{F}_{\mathrm{Tx}}^{\mathrm{T}}(\varphi) d \phi d \varphi,
$$


where $\boldsymbol{F}_{\mathrm{Rx}}(\phi)$ and $\boldsymbol{F}_{\mathrm{Tx}}(\varphi)$ are the beam gain matrix for receiver ( $\mathrm{Rx})$ and transmit antenna (Tx) on directions $\phi$ and $\varphi$, respectively. $\boldsymbol{H}(t ; \tau, \phi, \varphi)$ is the dual-polarized channel response matrix. The channel coefficient from $\mathrm{Tx} s$ to $\mathrm{Rx}$ element $u$ for cluster $n$ is given as [8]

$$
\begin{aligned}
& h_{u, s, n}(t ; \tau)= \\
& \sum_{m=1}^{M}\left[\begin{array}{l}
F_{\mathrm{Rx}, u, \mathrm{~V}}\left(\varphi_{n, m}\right) \\
F_{\mathrm{Rx}, u, \mathrm{H}}\left(\varphi_{n, m}\right)
\end{array}\right]\left[\begin{array}{cc}
\alpha_{n, m}^{V V} & \alpha_{n, m}^{V H} \\
\alpha_{n, m}^{H V} & \alpha_{n, m}^{H H}
\end{array}\right]\left[\begin{array}{l}
F_{\mathrm{Tx}, u, \mathrm{~V}}\left(\phi_{n, m}\right) \\
F_{\mathrm{Tx}, u, \mathrm{H}}\left(\phi_{n}, m\right)
\end{array}\right] \\
& \times \exp \left(j 2 \pi \lambda_{0}^{-1}\left(\bar{\varphi}_{n, m} \cdot \bar{r}_{\mathrm{Rx}, u}\right)\right) \exp \left(j 2 \pi \lambda_{0}^{-1}\left(\bar{\phi}_{n, m} \cdot \bar{r}_{\mathrm{Tx}, s}\right)\right) \\
& \times \exp \left(j 2 \pi v_{n, m} t\right) \delta\left(\tau-\tau_{n, m}\right),
\end{aligned}
$$

where $\alpha_{n, m}^{V V}$ and $\alpha_{n, m}^{V H}$ are the complex gains of vertical-tovertical and horizontal-to-vertical polarizations of ray $n, m$ respectively and $F_{\mathrm{Rx}, u, \mathrm{~V}}$ and $F_{\mathrm{Rx}, u, \mathrm{H}}$ are the field patterns for vertical and horizontal polarizations of antenna element $u$ respectively. Parameter $\lambda_{0}$ is the wave length of the carrier frequency, $\bar{\phi}_{n, m}$ is the angle of departure (AoD) unit vector, $\bar{\varphi}_{n, m}$ is the angle of arrival (AoA) unit vector, $\bar{r}_{\mathrm{Tx}, s}$ and $\bar{r}_{\mathrm{Rx}, u}$ are the location vectors of elements $s$ and $u$ respectively, and $v_{n, m}$ is the Doppler frequency of ray $n, m$. If polarization is not considered, the central matrix in the second line of (4) is replaced by a scalar $\alpha_{n, m}$ and only vertically polarized field pattern is considered. More details on the channel model can be found in [8].

The radiation antenna pattern in the azimuth angle direction [9] is given as

$$
A_{\mathrm{A}}(\theta)=-\min \left[12\left(\frac{\theta}{\theta_{3 \mathrm{~dB}}}\right)^{2}, A_{\mathrm{m}}\right],-180^{\circ} \leq \theta \leq 180^{\circ},
$$

where $\theta_{3 \mathrm{~dB}}$ is the $3 \mathrm{~dB}$ beamwidth which is $65^{\circ}$ for 3 -sector cell and $A_{\mathrm{m}}=30 \mathrm{~dB}$ is the maximum attenuation. In the elevation angle direction, the radiation antenna pattern is expressed as

$$
A_{\mathrm{E}}(\gamma)=-\min \left[12\left(\frac{\left(\gamma-90 \mathrm{deg}-\gamma_{\text {tilt }}\right)}{\gamma_{3 \mathrm{~dB}}}\right)^{2}, A_{\mathrm{m}}\right],
$$

where $\gamma_{3 \mathrm{~dB}}=65^{\circ}$ is the $3 \mathrm{~dB}$ vertical beamwidth and $0^{\circ} \leq$ $\gamma \leq 180^{\circ}$

\section{Antenna Array}

An illustration of the antenna coordination system and an incident wave is shown in Fig. 1.

The spatial displacement of the receive and transmit antenna elements and phase shifts among them is included in terms of $\exp \left(j 2 \pi \lambda_{0}^{-1}\left(\bar{\varphi}_{n, m} \cdot \bar{r}_{\mathrm{Rx}, u}\right)\right)$ and $\exp \left(j 2 \pi \lambda_{0}^{-1}\left(\bar{\phi}_{n, m} \cdot \bar{r}_{\mathrm{Tx}, s}\right)\right)$ in (4), where $\bar{r}_{\mathrm{Rx}, u}=\left[x_{u}, y_{u}, z_{u}\right]^{\mathrm{T}}$ is the location of the $u$ th antenna element. The unit position vector is

$$
\bar{\varphi}=[\cos \theta \cos \gamma, \sin \theta \cos \gamma, \sin \gamma]^{\mathrm{T}},
$$

where $\theta$ is the arrival azimuth angle and $\gamma$ is the arrival elevation angle. The phase delay of element $u$ can be calculated as

$$
\begin{aligned}
v_{(\gamma, \theta)} & =\bar{\varphi} \cdot \bar{r}_{\mathrm{Rx}, u} \\
& =\left(x_{u} \cos \theta \cos \gamma+y_{u} \sin \theta \cos \gamma+z_{u} \sin \gamma\right)
\end{aligned}
$$

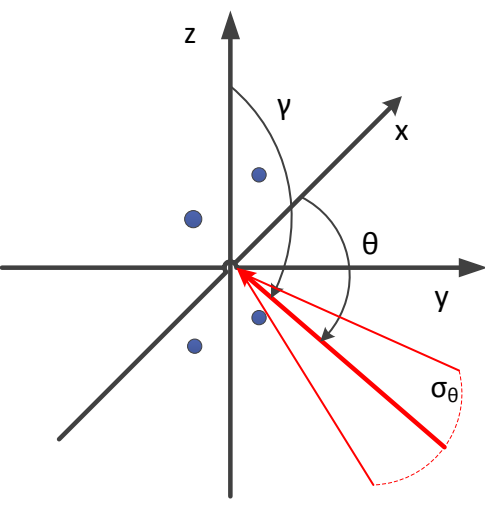

Fig. 1. An illustration of antenna coordination system.

\section{Spatial Correlation of ULA AND UPA}

In this section, the impact of the elevation domain in point-to-point, single user MIMO is studied. Closed-form expressions for the spatial correlation functions for 3D antenna arrays were derived in [4]. The elevation and azimuth angles were assumed uniformly distributed. The spatial correlation between the $u$ th and $v$ th array element is given by [2]

$$
\begin{aligned}
R_{s}(u, v) & =E\left\{v_{u}(\gamma, \theta) v_{v}^{*}(\gamma, \theta)\right\} \\
& =\int_{\gamma} \int_{\theta} v_{u}(\gamma, \theta) v_{v}^{*}(\gamma, \theta) p(\gamma, \theta) \sin \gamma d \gamma d \theta,
\end{aligned}
$$

where $p(\gamma, \theta)$ is the angular distribution function of the incoming plane wave. The closed-form approximation of the spatial correlation function between the $u$ th and $v$ th antenna elements with uniformly distributed $\gamma$ and $\theta$ can be found in [4].

The average correlation between the antenna elements in UPA and ULA as a function of the elevation or azimuth spreads are shown in Fig. 2. The central elevation and azimuth angles are $90^{\circ}$ and the angle spreads are uniformly distributed. The antenna spacing is $0.5 \lambda$. The changes in elevation spread do not have an impact on the ULA. As the elevation spread increases, the correlation in the UPA decreases. The increase in azimuth spread decreases the correlation in both the ULA and UPA.

The random capacity of a beamforming system can be written as [10]

$$
C=\log _{2}\left(1+N \frac{S N R}{2} \lambda_{s}\right),
$$

where $\lambda_{s}$ is the maximum singular value of $R_{s}$.

The capacity of a 16 transmit antenna beamforming system using (9) and (10) is illustrated in Fig. 3. The antenna spacing is $0.5 \lambda$. The capacity of the horizontal ULA does not change when the elevation spread increases but decreases when the azimuth spread increases. The result is opposite in the vertical ULA. The same result can be seen for the UPA as the capacity decreases with the increase in elevation spread but no change is observed when the azimuth spread increases. 


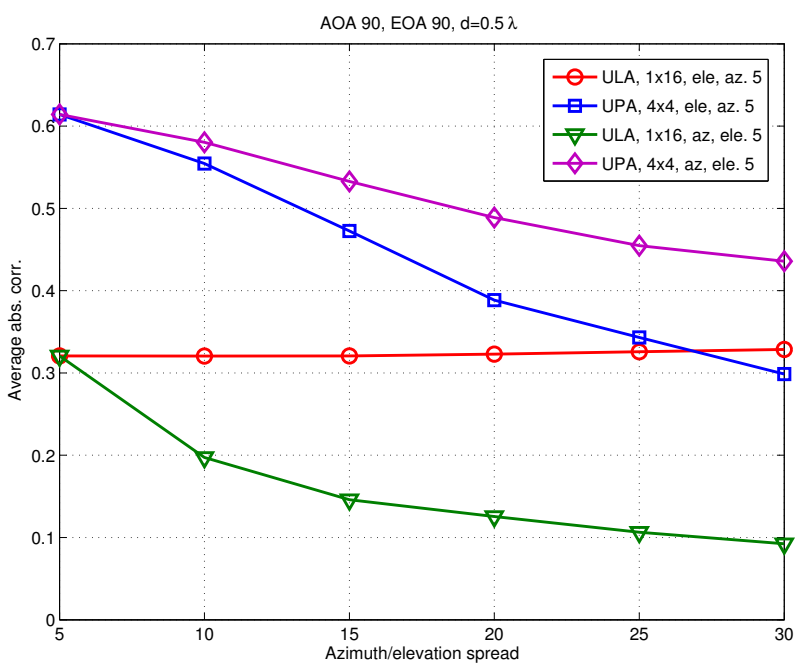

Fig. 2. Average correlation between the antenna elements.

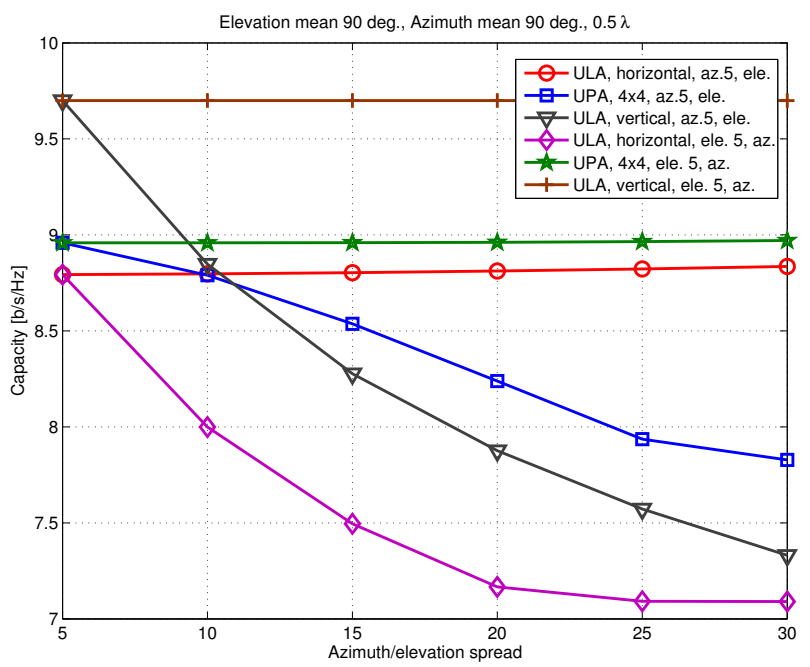

Fig. 3. Capacity of a beamforming system.

\section{Array Processing Algorithms}

The 3D MIMO from a system level point of view is studied via different beamforming techniques and multicell network simulations. Different beamforming weight calculation techniques were used when comparing the performances of the 3D antenna arrays. The optimal beamforming is compared to the codebook based beamforming. Separate beamforming weights for azimuth and elevation domains are also applied. The uplink power control and beamforming is performed similarly in all techniques.

Given the receiver beamformers $\boldsymbol{w}_{k, i}$ and the cooperation BS set $\pi_{k}$, the power control, BS cooperation reception and beamforming problem can be formulated as

$$
\begin{array}{ll}
\underset{\pi_{k}, p_{k}}{\operatorname{minimize}} & \sum_{k=1}^{K} p_{k} \\
\text { subject to } & \frac{\left|\sum_{i \in \pi_{k}} \boldsymbol{w}_{k, i}^{\mathrm{H}} \boldsymbol{h}_{k, i}\right|^{2} p_{k}}{\sum_{k^{\prime} \neq k}\left|\sum_{i \in \pi_{k}} \boldsymbol{w}_{k, i}^{\mathrm{H}} \boldsymbol{h}_{k^{\prime}, i}\right|^{2} p_{k^{\prime}}+\sum_{i \in \pi_{k}}\left\|\boldsymbol{w}_{k, i}\right\|^{2} \sigma_{i}^{2}} \geq \varphi_{k}, \\
& p_{k} \leq P_{k}
\end{array}
$$

where $|\cdot|$ denotes the absolute value, $\|\cdot\|$ the standard Euclidean vector norm, $\varphi_{k}$ is MS $k$ 's SINR requirement and $P_{k}$ is the maximum transmit power of MS $k$. The optimal beamforming vector is given by

$$
\begin{aligned}
& \left\{\boldsymbol{w}_{k, 1}, \boldsymbol{w}_{k, 2}, \cdots, \boldsymbol{w}_{k,\left|\pi_{k}\right|}\right\}= \\
& \arg \max _{\boldsymbol{w}_{k, i}} \frac{\left.\sum_{i \in \tilde{\pi}_{k}} \tilde{\boldsymbol{w}}_{k, i}^{\mathrm{H}} \boldsymbol{h}_{k, i}\right|^{2} \tilde{p}_{k}}{\sum_{k^{\prime} \neq k}\left|\sum_{i \in \tilde{\pi}_{k}} \tilde{\boldsymbol{w}}_{k, i}^{\mathrm{H}} \boldsymbol{h}_{k^{\prime}, i}\right|^{2} \tilde{p}_{k^{\prime}}+\sum_{i \in \tilde{\pi}_{k}}\left\|\tilde{\boldsymbol{w}}_{k, i}\right\|^{2} \sigma_{i}^{2}}
\end{aligned}
$$

The minimum total transmit power solution, the optimal receiving BSs set and the optimal beamforming vector can be found after iterative search, as long as the power vectors of the MSs are feasible. A more detailed description of the algorithm can be found in [11]. When assuming only one base station, i.e., no cooperation, the set $\pi_{k}$ includes only the serving base station and the index $i$ can be omitted.

For the baseline case, the optimal beamforming vectors are calculated as the MMSE filter and a general form is given as

$$
\boldsymbol{w}=\left(p \boldsymbol{h} \boldsymbol{h}^{H}+\sigma_{i}^{2} \boldsymbol{I}\right)^{-1} \boldsymbol{h} .
$$

For a simplified case, the beamforming vectors are taken from a codebook and the MS calculates the best beamforming vector. It is assumed that the MS knows the channel and it selects the beamforming vector based on channel capacity. The codebook for the 16 transmit and one receive antenna case was extended from the codebook for 8 transmit antennas in [12]. The codebook is given in Table I.

Different weighting vectors can be applied to the azimuth and elevation dimensions. For the case of 16 transmit antennas in the base station, a $4 \times 1$ weighting vector is used for both dimensions. The azimuthal weighting vectors are then chosen from the LTE codebook [13]. Two methods for finding the elevation weighting vectors were used. In the first method, a set of fixed elevation weighting vectors were applied. The coefficients for the weighting vectors $\boldsymbol{w}_{V}=\left[w_{V, 1}, w_{V, 2}, \ldots, w_{V, n_{V}}\right]$ were given as [14]

$$
w_{V, c}=\frac{1}{\sqrt{n_{V}}}\left[j 2 \pi(c-1) \frac{d_{V}}{\lambda} \cos \check{\gamma}\right], c=1, \ldots, n_{V},
$$

where $n_{V}$ is the number of antennas placed in the elevation dimension, $d_{V}$ is the antenna spacing in elevation, $\lambda$ is the wave length of carrier frequency and $\check{\gamma}$ is the angle of the steering beam.

In the second method, the elevation weighting vector is calculated as the eigenvector corresponding to the largest 


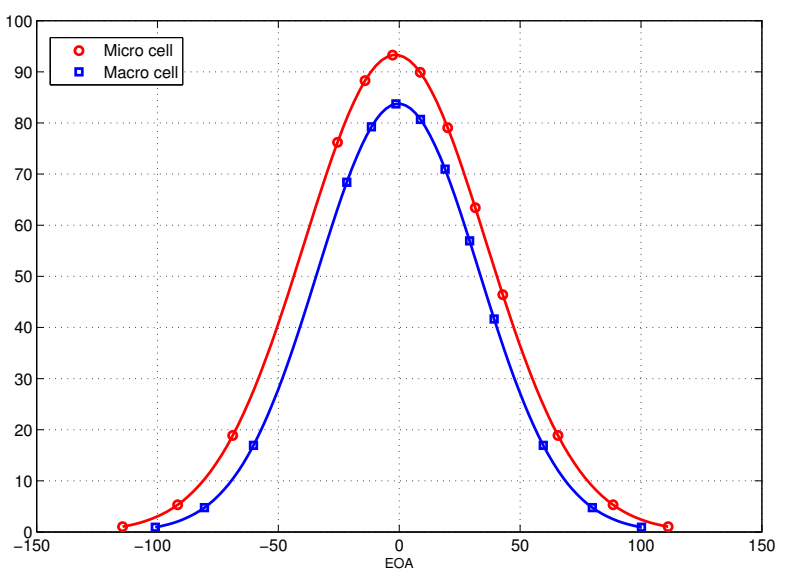

Fig. 5. Distribution of elevation angles of arrival for Urban Macro/Micro cell scenarios.

An 8 degree down tilt angle is used. Results with SINR constraint $0 \mathrm{~dB}$ are plotted. The performances of the ULA and UPA with optimal beamforming differ significantly. If the codebook based beamforming is used, the performance degradation compared to the optimal case is significant. If separate weighting vectors for the elevation and azimuth domains are used, the link is not feasible with higher SINR constraints and the maximum transmit power constraint is exceeded. With the SINR constraint of $0 \mathrm{~dB}$, the eigenvector elevation weighting method has the best performance of all the suboptimal methods in the UPA case. It also performs similarly as the codebook based ULA. This is due to the fact that part of the elevation weight vectors are calculated from the channel matrices as in the optimal beamforming case.

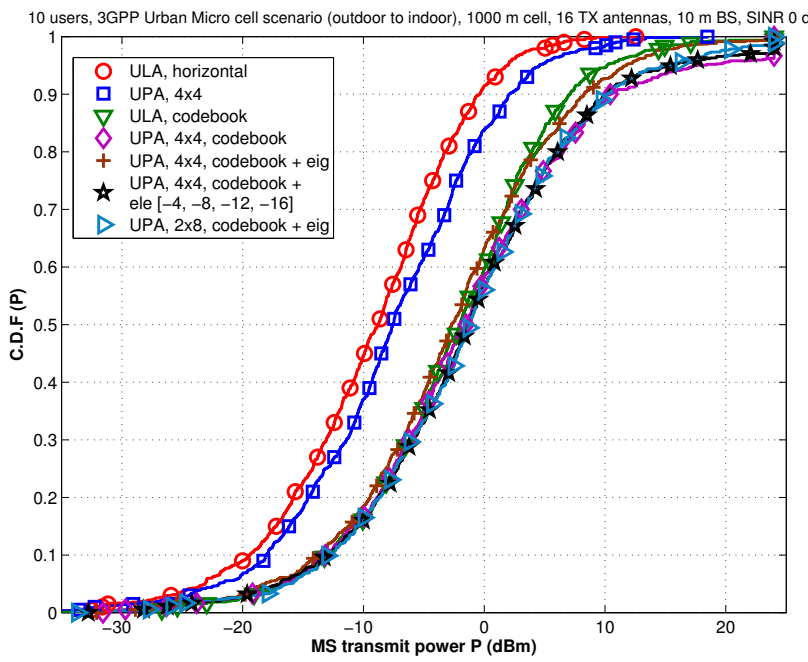

Fig. 6. Transmit power of ULA and UPA with 10 users and Urban Micro cell scenario.

The ULA and UPA performance in the Urban Macro cell scenario are presented in Fig. 7. The down tilt angle is 4 degrees. The differences between the beamforming methods and the antenna arrays are very similar to those in Fig. 6 even though the performance in the Macro cell is better in general. When codebook based beamforming is used, the ULA still outperforms the UPA. Eigenvector based elevation weighting brings performance gain also in the Urban Macro cell scenario.

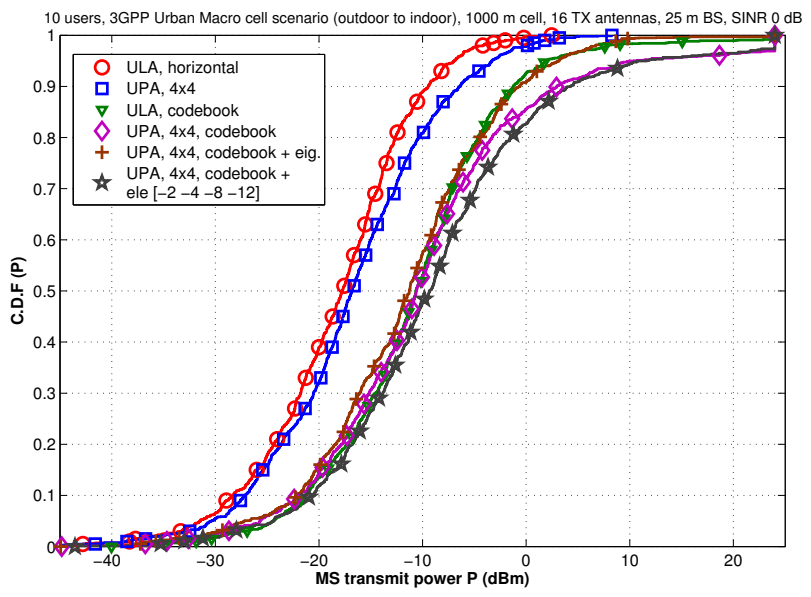

Fig. 7. Transmit power of ULA and UPA with 10 users and Urban Macro cell scenario.

\section{COMPleXity ESTIMATIONS}

The complexity of the different array processing algorithms presented in Section IV is given here in numbers of operations. The number of operations for each beamforming weight calculation per user in a 16 transmit antenna case is given in Table IV. In the optimal beamforming case, where the beamforming weight vector is given as (12), an $N_{i} \times N_{i}$ linear system needs to be solved. In this case, a $16 \times 16$ matrix inversion needs to be calculated for every user. In the codebook based method, the weight matrix is suggested by the user and no processing is required at the BS. However, this increases the calculation burden in the user equipment. When the azimuthal codebook is suggested by the user equipment, the BS calculates the best elevation vector, resulting in a low amount of operations. With the eigenvector based elevation weights, an eigenvalue decomposition for a $4 \times 4$ matrix is calculated at the BS, assuming 16 transmit antennas. This increases the complexity slightly compared to the fixed elevation weights.

TABLE IV

REQUIRED NUMBER OF OPERATIONS FOR BEAMFORMING WEIGHT CALCULATION

\begin{tabular}{|l|l|}
\hline Method & Operations \\
\hline Optimal beamforming & 10260 \\
\hline Codebook based & - \\
\hline Codebook az., fixed elevation & 88 \\
\hline Codebook az., eigenvector elevation & 286 \\
\hline
\end{tabular}




\section{CONCLUSiOnS}

Comparisons between the ULA and UPA were made in terms of their spatial correlation and theoretical beamforming capacity. Network simulations were then performed with the latest 3D channel models to obtain insight into their performance differences. Different array processing algorithms were also utilized.

The ULA was found to outperform the UPA in all the simulated scenarios with optimal beamforming. The results match those given by the theoretical beamforming capacity. A slight increase in performance can be obtained by dividing the processing into azimuth and elevation domains compared to the fixed codebook case. This also gives significant complexity savings in calculating the beamforming weights compared to the optimal beamforming case.

\section{REFERENCES}

[1] J. Koppenborg, H. Halbauer, S. Saur, and C. Hoek, "3D beamforming trials with an active antenna array," in Proc. Int. ITG Works. on Smart Antennas (WSA), Dresden, Germany, Mar. 7-8 2012, pp. 110-114.

[2] S. K. Yong and J. Thompson, "A three-dimensional spatial fading correlation model for uniform rectangular arrays," IEEE Antennas Wireless Propagat. Lett., vol. 2, no. 1, pp. 182-185, 2003.

[3] J. Raj, S. Arokiasamy, N. Vikram, and J. Schoebel, "Spatial correlation and MIMO capacity of uniform rectangular dipole arrays," IEEE Antennas Wireless Propagat. Lett., vol. 7, pp. 97-100, May 2008.

[4] J. Lee and C. Cheng, "The spatial correlation characteristics of 3-D antenna array systems," in Int. Midwest Symp. on Circuits and Systems (MWSCAS), Seoul, South Korea, Aug. 7-10 2011, pp. 1-4.

[5] M. Narandzic, M. Kaske, C. Schneider, M. Milojevic, M. Landmann, G. Sommerkorn, and R. Thoma, "3D-antenna array model for ISTWINNER channel simulations," in Proc. IEEE Veh. Technol. Conf., Dublin, Ireland, Apr. 22-25 2007, pp. 319-323.

[6] 3rd Generation Partnership Project (3GPP); Technical Specification Group Radio Access Network, "Study on 3D channel model for LTE (Release 12));," 3rd Generation Partnership Project (3GPP), Tech. Rep., 2014.

[7] X. Lu, A. Tölli, O. Piirainen, M. Juntti, and W. Li, "Comparison of antenna arrays in a 3-D multiuser multicell network," in Proc. IEEE Int. Conf. Commun., Kyoto, Japan, Jun. 5-9 2011, pp. 1-6.

[8] P. Kyösti, "Winner II channel models, IST-4-027756 WINNER II, D1.1.2 V1.1,” WINNER Project, Tech. Rep., Sep. 2007.

[9] "Guidelines for evaluation of radio interface technologies for IMTAdvanced," International Telecommunication Union, Tech. Rep.

[10] B. Friedlander and S. Scherzer, "Beamforming versus transmit diversity in the downlink of a cellular communications system," IEEE Trans. Veh. Technol., vol. 53, no. 4, pp. 1023-1034, Jul. 2004.

[11] X. Lu, W. Li, A. Tölli, M. Juntti, E. Kunnari, and O. Piirainen, "Joint power control, receiver beamforming and adaptive multi base station coordination for uplink wireless communications," in Proc. IEEE Int. Symp. Pers., Indoor, Mobile Radio Commun., Workshop on Wireless Distributed Networks, Sep. 2010, pp. 446 - 450.

[12] Motorola, "DL codebook design for 8 TX antennas for LTE-A, 3GPP TSG RAN1-56," 3rd Generation Partnership Project (3GPP), Tech. Rep., 2009.

[13] 3rd Generation Partnership Project (3GPP); Technical Specification Group Radio Access Network, "Evolved universal terrestrial radio access E-UTRA; physical channels and modulation (release 8) TS 36.211 (version 8.5.0)," Tech. Rep., 2008.

[14] Y. Song, X. Yun, S. Nagata, and L. Chen, "Investigation on elevation beamforming for future LTE-advanced," in Proc. IEEE Int. Conf. Commun. Workshops, Budapest, Hungary, Jun.9-13 2013, pp. 106-110.

[15] T. Thomas and F. Vook, "Transparent user-specific 3D MIMO in FDD using beamspace methods," in Proc. IEEE Global Telecommun. Conf., Anaheim, USA, Dec.3-7 2012, pp. 4618-4623.

[16] T. Hytönen, "Optimal wrap-around network simulation," Helsinki university of technology, Espoo, Finland, Helsinki university of technology institute of mathematics research reports, 2001. 